\title{
Propuesta metodológica para el estudio de MACRORRestos de BRIOFITAS EN TURBERAS
}

\author{
ADOLFINA SAVORETTII*, DAMIÁN ANDRÉS FERNÁNDEZ ${ }^{2 *}$, ARI IGLESIAS $^{3^{*}} \mathrm{y}$ \\ JUAN FEDERICO PONCE ${ }^{4,5^{*}}$
}

\begin{abstract}
Summary: Methodological contributions for the analysis of bryophyte macroremains in peatlands. This contribution describes in detail the methods used in the processing of samples collected in peats for paleobryofloristic studies based on Quaternary plant macrorests. The sample processing methods proposed here ensure that preserved plant remains are recovered with minimal damage, making possible quantitative assessment of different types of remains of each taxon/morphotype for comparative studies of taphonomy and for paleoecological interpretations. Digitalization of sample images is proposed to allow for easy comparisons of macrorests between aliquots, sample sections and peat samples from different regions, and for taxonomic identifications of some of the material. These methods provide for repeatability, which is necessary for comparisons between analyses of different investigators.
\end{abstract}

Key words: Bryophytes, method, plant macroremains, Quaternary.

Resumen: En esta contribución se dan a conocer las técnicas empleadas para el procesamiento de testigos provenientes de turberas, con el fin de abordar estudios paleobrioflorísticos sobre la base de macrorrestos del Cuaternario. El procesamiento propuesto procura que los restos preservados sean dañados en la menor medida posible y realizar un conteo de los diferentes tipos de fragmentos de cada uno de los taxones/morfotipos con el objetivo de realizar estudios comparativos de la tafonomía y con ello enriquecer las interpretaciones paleoecológicas y la comprensión del desarrollo de la turbera. Se propone adoptar la digitalización de imágenes de las muestras, permitiendo comparar de forma simple los macrorrestos entre las alícuotas, entre las secciones del testigo y entre testigos de turberas de cualquier región, e incluso poder realizar determinaciones y cuantificaciones de parte de su material. Esta metodología ofrece una repetitividad necesaria para la comparación entre análisis de diferentes investigadores.

Palabras clave: Briofitas, Cuaternario, macrorrestos vegetales, metodología.

\footnotetext{
${ }^{1}$ Instituto de Botánica Darwinion, Labardén 200, San Isidro (1642), Buenos Aires Argentina. ANCEFN

2 Sección Paleopalinología, MACN "B. Rivadavia”, Ángel Gallardo 470 (C1405DJR) C.A.B.A., Argentina

${ }^{3}$ Instituto de Investigaciones en Biodiversidad y Medioambiente. Quintral 1250 - San Carlos de Bariloche 8400 - Río Negro - Argentina

${ }^{4}$ Centro Austral de Investigaciones Científicas, CADIC. Bernardo Houssay 200, Ushuaia (9410), Tierra del Fuego, Argentina

${ }^{5}$ Universidad Nacional de Tierra del Fuego, Instituto de Ciencias Polares, Ambiente y Recursos Naturales, Av. Los Ñires 2382, Ushuaia (9410), Tierra del Fuego, Argentina

*Consejo Nacional de Investigaciones Científicas y Técnicas (CONICET), Argentina
} 


\section{INTRODUCCIÓN}

Se consideran macrorrestos vegetales a aquellos restos que aunque pequeños, son visibles a simple vista y poseen un tamaño medio de 0.5 a 2 milímetros (Dickson, 1986; Birks, 2007; Mauquoy et al., 2010). El uso de macrorrestos vegetales en investigaciones paleoecológicas comenzó en el siglo XIX en Europa, con anterioridad al desarrollo de los análisis polínicos (Birks, 2001). A partir de la década de 1920, al expandirse las metodologías polínicas, el uso de macrorrestos vegetales declinó. Cuatro décadas más tarde, al revelarse ciertas limitaciones de los análisis de palinomorfos, estas dos técnicas se establecieron como complementarias (Dickson, 1986; Janssens, 1990; Birks, 2001; Jakab et al., 2004).

Numerosos estudios de macrorrestos de briofitas se han llevado a cabo en depósitos de turberas de Europa y Norte América (e.g. Miller, 1980; Janssens, 1983; Barber, 1993). En el Hemisferio Sur, son menos los trabajos utilizando macrorrestos de briofitas en turberas, pero algunos han presentado excelente estado de preservación tanto en áreas continentales (Mauquoy et al., 2004; Markgraf \& Huber, 2010; Chambers et al., 2014) como en islas circum-antárticas (Van der Putten et al., 2004, 2008, 2009).

Las turberas son los únicos ecosistemas cuyas comunidades vegetales dejan in situ un registro detallado de su propia historia (Barber, 1993). La acumulación de macrofósiles ocurre a medida que los restos vegetales van quedando sepultados desde la porción superior, aeróbica de la turbera (acrotelm), a la porción inferior anaeróbica (catatelm) (Ingram, 1978; Vitt \& Wieder, 2009; Iturraspe, 2010). En general, se asocia al género Sphagnum como la única briofita formadora de turberas, sin embargo la flora briofítica de éstas puede ser muy diversa (Janssens, 1983, 1987; Dickson, 1986; Larraín, 2015) e incluso son varios los géneros que pueden jugar un rol muy importante en la composición de las turberas (Dickson, 1986; Vitt \& Luth, 2017).

A pesar de los cambios tafonómicos que los restos vegetales sufren durante el proceso de fosilización en las turberas (Clymo \& Hayward, 1982), las briofitas (especialmente los musgos) son los macrofósiles más comunes en los depósitos del Periodo Cuaternario (Miller, 1983; Janssens,
1990). Asimismo, dependiendo de su preservación, los macrorrestos pueden ser asignados a géneros o especies actuales, lo que hace a las briofitas excelentes indicadores paleoecológicos (Janssens, 1990; Barber, 1993).

A diferencia del procesamiento estandarizado para el análisis palinológico de sedimentos, la metodología para el estudio de macrorrestos es más heterogénea (De Vleeschouwer et al., 2010). Esto puede generar dificultades y dudas en la toma de decisiones por parte del investigador que se enfrenta con la metodología a aplicar. Por otra parte, los estudios de análisis de macrorrestos generalmente no presentan imágenes que ilustren la identificación de los taxones, lo cual es de gran importancia para posibilitar las comparaciones entre los hallazgos, así como posibilitar una primera identificación de los macrorrestos previo a la consulta del material de referencia, como propone Birks (2017), para el caso de las semillas.

El objetivo principal del presente trabajo es brindar, en forma detallada, una nueva propuesta metodológica con los diferentes pasos a seguir en el laboratorio para el procesamiento y estudio de muestras de testigos de turberas en base a macrorrestos del Cuaternario. La propuesta tiene el fin de unificar criterios de trabajos previos, para que al ser aplicadas puedan obtenerse resultados de macrorrestos vegetales comparables y replicables. Las técnicas presentadas han sido aplicadas con éxito en una turbera de Sphagnum localizada en la costa norte de Canal Beagle, Tierra del Fuego (Savoretti et al., 2016). Sobre la base de este análisis se ha desarrollado la metodología aquí propuesta.

Este aporte incorpora: (1) un modo de cuantificación de macrofósiles de briofitas que combina diferentes aspectos de propuestas previas, (2) la creación de un registro de los diferentes taxones de briofitas con énfasis en la disminución de la fragmentación (multiplicación) de los macrorrestos durante el proceso y (3) manteniendo un registro (backup) del material analizado.

\section{Materiales Y MÉtodos}

La metodología aquí propuesta ha sido empleada para el estudio de la evolución de la vegetación briológica de la turbera Harberton I (Heusser, 
1990), ubicada en la provincia de Tierra del Fuego, Argentina. Esta turbera se localiza $700 \mathrm{~m}$ al norte de la costa norte del Canal Beagle, en el interior de un campo de geoformas subglaciales denominadas drumlins. Este conjunto de geoformas se habría originado por acción del Paleoglaciar Beagle durante el Último Máximo Glacial, 24.000 años AP (Rabassa, 2008).

La sección fósil estudiada (Savoretti et al., 2016) se extrajo con una sonda tipo rusa, con sacatestigo de $50 \mathrm{~cm}$ de longitud. Dicha sección presenta $10,5 \mathrm{~m}$ de longitud y corresponde a la máxima profundidad alcanzada. De este testigo se han analizado los restos vegetales de 50 submuestras. Dichas submuestras corresponden tanto a la porción basal (minerotrófica) como a la superior (ombrotrófica) de la turbera (Heusser, 1990). Siendo el objetivo del presente trabajo exponer la metodología aplicada para tal fin, solo se desarrollan ejemplos ilustrativos para la compresión del método propuesto.

\section{RESULTADOS}

Los pasos para llevar a cabo un estudio paleoflorístico del Cuaternario sobre la base de macrorrestos de briofitas son: (I) obtención del testigo, (II) submuestreo del testigo, (III) procesamiento físico de las submuestras, (IV) digitalización del registro de macrorrestos (V) cuantificación de macrorrestos, (VI) determinación de macrorrestos (VII) análisis de datos y representación de resultados. En forma paralela a los pasos mencionados, se elaboran colecciones de referencia, tanto del material actual (de superficie) como del fósil.

Para los pasos de obtención y submuestreo del testigo (pasos I y II) se ha seguido las recomendaciones de trabajos previos que han sido tratados detalladamente en De Vleeschouwer et al. (2010). En dicho trabajo se propone que el sitio ideal para extraer un testigo de una turbera ombrotrófica es en la interfase montículo-depresión, ya que de allí se obtiene el registro estratigráfico más sensible a los cambios del contenido hídrico y por lo tanto de la vegetación; se sugieren diferentes herramientas para la obtención del testigo, se recomienda emplear la técnica de dos pozos paralelos para disminuir la contaminación de la turba extraída; se mencionan, además, todas las precauciones de limpieza que deben tenerse en el proceso de obtención de las submuestras. Se recomienda la consulta del protocolo propuesto por De Vleeschouwer et al. (2010), previamente a comenzar un estudio paleoambiental en turberas.

En cuanto a la determinación de macrorrestos, análisis y representación de datos (pasos VI y VII) han sido abordados en detalle por Dickson (1986), Janssens (1987), Birks (2001, 2017). La representación de los resultados puede hacerse de diversas maneras, como ser en forma de listas de presencia/ausencia de los diferentes taxa, o con números de abundancia para cada uno de éstos. Otro modo propuesto se basa en los diagramas de concentración: número de restos en un volumen constante de sedimento calculado a partir de los conteos en volúmenes de sedimento conocidos (Birks, 2017). La herramienta informática más frecuentemente utilizada para graficar diagramas de macrorrestos es TILIA.GRAPH.

En el presente trabajo, se tratan en forma más detallada los pasos del procesamiento de las submuestras (paso III), digitalización del registro de macrorrestos (paso IV) y cuantificación de macrorrestos (paso V); así como se comentan detalles menores para la determinación de los macrorrestos de briofitas (paso VI).

\section{Procesamiento de las submuestras}

Una vez realizada la obtención del testigo (paso I) y seleccionados los niveles a analizar, se realiza el procesamiento de las submuestras de la siguiente manera:

Obtención del material a procesar. Se divide completamente el testigo en submuestras representativas de cada nivel (para el caso aquí presentado, cada $2,5 \mathrm{~cm}$ ) y se colocan las submuestras en bolsas herméticas con los rótulos correspondientes; se almacenan en frío hasta volver a utilizarse. Posteriormente se obtiene, de cada submuestra a estudiar, una porción de la misma equivalente a un volumen de $5 \mathrm{~cm}^{3}$ (Van der Putten et al., 2009; De Vleeschouwer et al., 2010; Mauquoy et al., 2010), extrayéndose con pinzas o cuchillo, y se coloca en un recipiente gradado plástico de $5 \mathrm{~cm}^{3}$ (Fig. 1, C).

Lavado. Para el lavado de las submuestras se utiliza una cubeta plástica con agua cubriendo la mitad de su volumen. En ésta se coloca un 
tamiz, malla de $250 \mu \mathrm{m}$ (Mauquoy et al., 2010), y sobre éste se vierten los $5 \mathrm{~cm}^{3}$ de la submuestra. Manteniendo el tamiz en el agua, se lo agita suavemente. Para facilitar la disgregación del material se recomienda la utilización de pinzas y realizar el trabajo bajo lupa (aumento de $5 \mathrm{x}$ ), con el fin de observar si el material se daña durante este proceso (Fig. 1, A). Se cambia el agua de la cubeta cuantas veces sea necesario para que la mayor parte del sedimento fino sea eliminado (generalmente de una a tres veces).

Separación del material a analizar. Se recupera el material del tamiz y se lo coloca en un frasco graduado, de $100 \mathrm{~cm}^{3}$. Es aconsejable, utilizar una pizeta, y hacer correr el agua por el tamiz en su cara inferior, de modo que los macrorrestos se deslicen hacia el recipiente sin romperse. Así, se tienen los $5 \mathrm{~cm}^{3}$ de material en $75 \mathrm{~cm}^{3}$ de agua destilada (aproximadamente, según la composición de cada submuestra).

Conservación y almacenamiento. Se agregan 2-3 gotas de $\mathrm{HCl}$ al $10 \%$ (Janssens, 1990; Barber, 1993) y se guarda en el refrigerador o cámara fría a $4{ }^{\circ} \mathrm{C}$. De esta manera se intenta evitar la proliferación de descomponedores que puedan afectar el material.
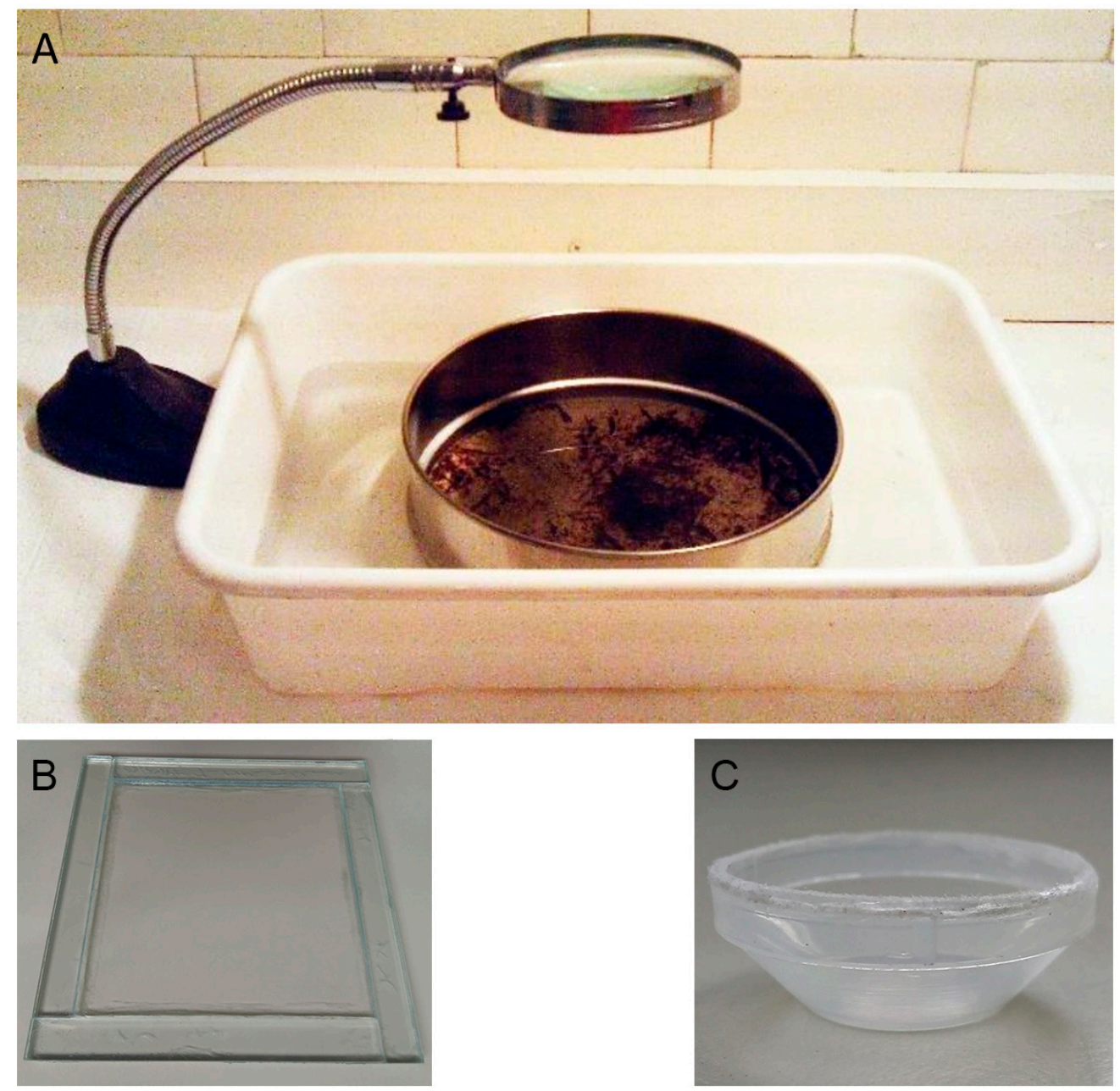

Fig. 1. Herramientas de laboratorio utilizadas en el procesamiento y en la obtención de imágenes digitales. (A). Tamiz, batea y lupa empleados para lavar y disgregar el material a analizar. (B). Recipiente plano de vidrio de 16 × $16 \mathrm{~cm}$ internos, utilizado para el escaneo de la submuestra. (C) Recipiente de $5 \mathrm{~cm}^{3}$ utilizado para submuestrear el testigo. 


\section{A. Savoretti et al. - Metodología para macrorrestos de briofitas de turberas}

\section{Digitalización de la muestra}

Este paso tiene como objetivo la obtención de imágenes digitales generales de cada submuestra a analizar y de las cinco alícuotas seleccionadas para contar (ver más adelante).

\section{Obtención de imágenes}

Imagen general de cada muestra. Se utiliza un recipiente de vidrio de $16 \times 16 \mathrm{~cm}$ internos y $3 \mathrm{~mm}$ de profundidad (Fig. 1, B), ya que en éste área los $80 \mathrm{~cm}^{3}$ de submuestra quedan homogéneamente esparcidos en una monocapa de material vegetal. Se ubica el recipiente, junto con una escala y rótulo, en un escáner plano, y se vierte el contenido del frasco. Se esparce y se escanean con una resolución mínima de 800 ppp (pixeles por pulgada) (Fig. 2, A y C). Con dicha resolución es posible la identificación de fragmentos de porte muy pequeño, como se ejemplifica en la Figura 2 con fragmentos de Sphagnum magellanicum, dominante en turberas ombrotróficas y con fragmentos de Amblystegiaceae, importante
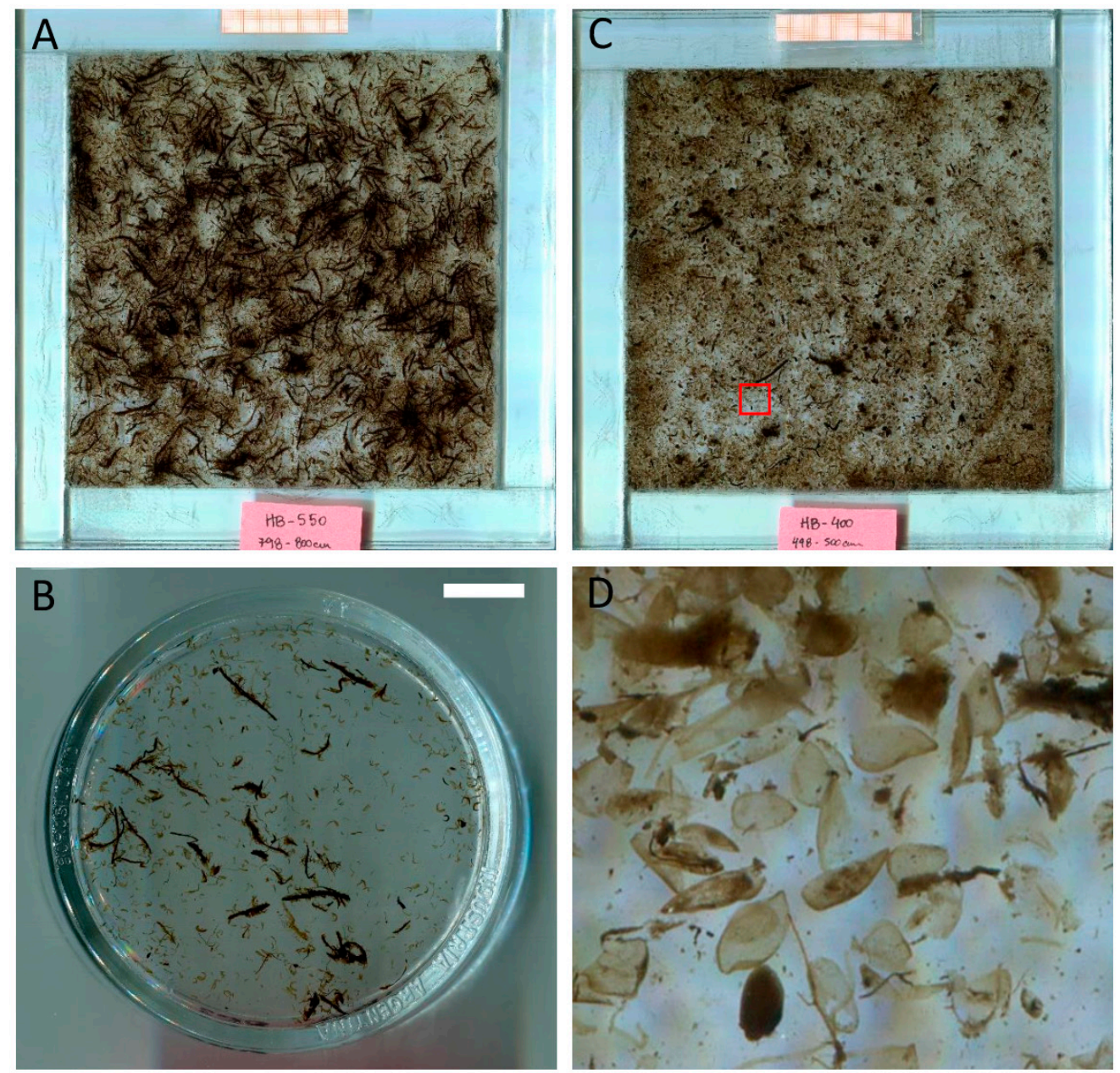

Fig. 2. Ejemplo de digitalización de submuestras (repositorio digital) provenientes de la turbera Haberton I ( $\mathrm{HBI}$ ), de diferente composición briofítica. (A) Escaneo de submuestra de $5 \mathrm{~cm}^{3}$ en $75 \mathrm{~cm}^{3}$ de agua, briofita (Amblystegiaceae) dominante en primeros estadios de la turbera. (B). Escaneo de una alícuota de la submuestra mostrada en (A). (C) Escaneo de submuestra de $5 \mathrm{~cm}^{3}$ en $75 \mathrm{~cm}^{3}$ de agua, briofita dominante Sphagnum magellanicum. (D) Detalle de la imagen 2(C), en donde se muestra la definición del escaneo, que posibilita una observación detallada de los macrorrestos. 
componente en ambientes minerotróficos (Ochyra \& Matteri, 2001).

Imágenes de alícuotas seleccionadas. Con una pipeta Pasteur de $3 \mathrm{ml}$ se toman cinco submuestras de cinco puntos diferentes del recipiente, los cuales representen los materiales de los cuatro vértices y el centro del mismo. Cada pipeteo se coloca en una cápsula de Petri (5 $\mathrm{cm}$ de diámetro), disponiéndose el material en una monocapa. Cada una de las cinco cápsulas con material se escanea (Fig. 2B), junto a escala y respectiva referencia. Es importante que los restos no estén suspendidos en el agua al momento de ser escaneados, ya que de lo contrario las imágenes que se obtienen no son claras y deben descartarse. Se recomienda guardar nuevamente la submuestra, llevando el material a un frasco desde el recipiente plano de vidrio, utilizando un embudo o bandeja.

\section{Cuantificación de los macrorrestos}

El material de las cinco alícuotas, dispuestas en las cápsulas de Petri (descripto en el paso previo), representa el total a analizar por muestra original. Este volumen, se considera representativo del contenido de macrorrestos de la submuestra total (5 $\mathrm{cm}^{3}$ iniciales), en base a esfuerzo de muestreo. Cada cápsula con material se observa con microscopio estereoscópico, con un aumento de 20x. Se consideran para el conteo todos los fragmentos potencialmente determinables, ya sean hojas enteras o fragmentos de estas, tallos con y sin hojas en conexión y esporofitos. En el caso de macrorrestos de Sphagnum, se discrimina entre hojas sueltas de ramas y hojas sueltas del tallo, ya que estas últimas son de relevancia taxonómica (Anderson et al., 2009). A medida que se realiza el conteo, se separan los macrorrestos en frascos independientes (de 10 $\mathrm{cm}^{3}$ ) con agua destilada y 2-3 gotas de $\mathrm{HCl} 10 \%$, rotulados según su determinación.

Al terminar el conteo, se observa la imagen digital general en búsqueda de morfotipos poco frecuentes, que no hayan sido observados en las alícuotas inspeccionadas. De encontrarse, se incluyen en el conteo con baja frecuencia.

Durante el procesamiento se anota en una planilla la presencia y cantidades (frecuencias) de cada taxón/morfotipo de macrorresto por cada cápsula de Petri por separado. Al finalizar se realiza una suma acumulativa para la muestra total (Tabla 1).
En el caso que una submuestra este representada en su mayoría por un único taxón, y que los fragmentos de este sean muy abundantes (cientos por alícuota) puede considerarse la alternativa de realizar el conteo de una sola alícuota, en términos de disminuir significativamente el esfuerzo de trabajo.

\section{Determinación de los macrorrestos}

De todos los macrorrestos separados por nivel analizado y por taxón/morfotipo designado en una primera inspección, se seleccionan aquellos que presentan mejor estado de conservación y se procede a realizar preparados para ser analizados bajo el microscopio óptico, para corroborar su asignación taxonómica. Para esto los macrorrestos pueden tratarse al igual que a las briofitas actuales (Schofield, 1985). Es importante evitar que el material se deshidrate, porque de este modo se vuelve frágil y tiende a desintegrarse (Janssens, 1988), particularmente los fragmentos muy lábiles como los del género Sphagnum.

Es necesario trabajar con bibliografía de floras actuales e imprescindible consultar colecciones de referencia para lograr asignaciones taxonómicas confiables, tanto de macrorrestos como de material actual del área de estudio; así como, consultar material de herbario (Birks, 2017).

\section{Discusión}

En la Tabla 2 se ha comparado la metodología planteada con propuestas anteriores. Dichos trabajos fueron elegidos por desarrollar metodologías innovadoras al momento de darse a conocer, y por haber sido posteriormente adoptadas por otros investigadores. Diferentes aspectos metodológicos han sido comparados.

En cuanto al volumen de la submuestra a analizar, se encuentra relativamente estandarizado en torno a los $5 \mathrm{~cm}^{3}$. Siendo una excepción el mayor volumen (50-200 ml) propuesto por Janssens (1983), pionero en el desarrollo cuantitativo de briofitas. En dicha propuesta el tiempo requerido para el análisis de tal volumen hace al método poco eficiente.

La obtención de la submuestra calculando su volumen por desplazamiento de agua es más laboriosa que la utilización de un recipiente plástico de volumen conocido y particularmente de $5 \mathrm{~cm}^{3}$, como se propone 
A. Savoretti et al. - Metodología para macrorrestos de briofitas de turberas

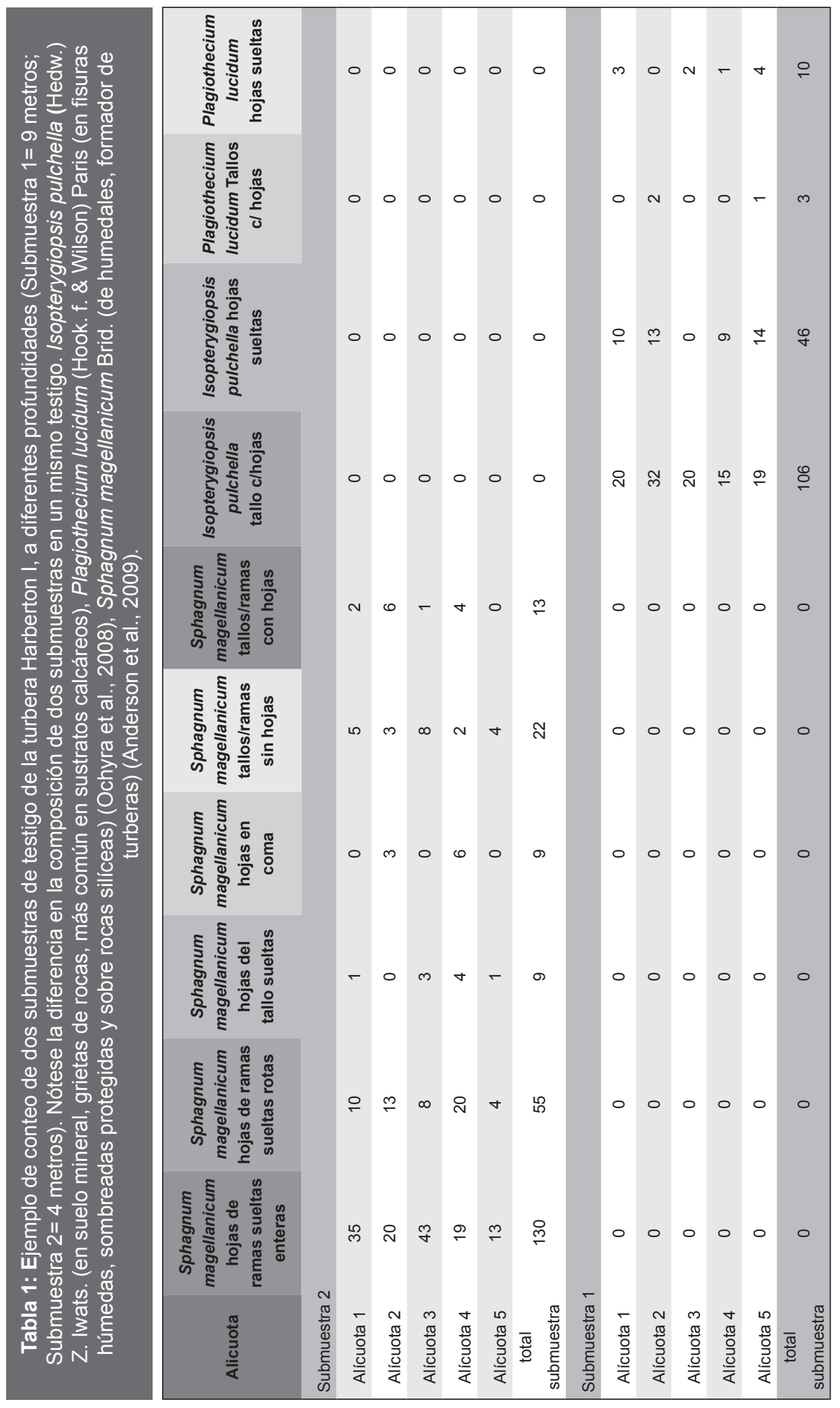


Tabla 2: Comparación de metodologías de procesamiento de macrorrestos vegetales. NE=No especificado; "no recomendado por ser destructivo (Janssens, 1990).

\begin{tabular}{|c|c|c|c|c|c|}
\hline & $\begin{array}{c}\text { Walker \& } \\
\text { Walker, } 1961\end{array}$ & Janssens, 1983 & Barber, 1993 & $\begin{array}{c}\text { Mauquoy et } \\
\text { al., } 2010\end{array}$ & Este trabajo \\
\hline $\begin{array}{c}\text { Volumen de } \\
\text { submuestra } \\
\text { a analizar }\end{array}$ & $\begin{array}{l}\text { Lámina de } 1 \\
\text { cm ancho }\end{array}$ & 50 a $200 \mathrm{ml}$ & $\begin{array}{l}\text { Lámina de } 1 \\
\text { cm }\left(4 \mathrm{~cm}^{3}\right)\end{array}$ & $5 \mathrm{~cm}^{3}$ & $5 \mathrm{~cm}^{3}$ \\
\hline $\begin{array}{l}\text { Obtención de la } \\
\text { submuestra }\end{array}$ & NE & $\begin{array}{c}\text { Desplazamiento } \\
\text { de agua }\end{array}$ & NE & $\mathrm{NE}$ & $\begin{array}{l}\text { Utilizando } \\
\text { recipiente } \\
\text { de } 5 \mathrm{~cm}^{3}\end{array}$ \\
\hline $\begin{array}{l}\text { Tratamiento } \\
\text { químico para } \\
\text { disgregar } \\
\text { submuestra }\end{array}$ & $\begin{array}{c}\text { Maceración } \\
\text { con soda } \\
\text { caustica al } 2 \%\end{array}$ & $\begin{array}{c}72 \text { horas en } \\
\text { agua con } \mathrm{KOH} \text { o } \\
\text { en } \mathrm{HCl} \text { diluído* }\end{array}$ & NE & $\begin{array}{c}5 \% \mathrm{KOH} / \\
\mathrm{NaOH}(35-40 \\
\mathrm{min}) \text { con calor }\end{array}$ & $\begin{array}{c}\text { Sin tratamiendo } \\
\text { químico }\end{array}$ \\
\hline Tamaño de malla & $100 \mu \mathrm{m}$ & $420 \mu \mathrm{m}$ & $125 \mu \mathrm{m}$ & $100-125 \mu \mathrm{m}$ & $250 \mu \mathrm{m}$ \\
\hline Modo de tamizado & NE & NE & $\begin{array}{l}\text { Chorros } \\
\text { fuertes de } \\
\text { agua }\end{array}$ & $\begin{array}{c}\text { Pizeta con agua } \\
\text { destilada }\end{array}$ & $\begin{array}{c}\text { En batea con } \\
\text { cambios de agua }\end{array}$ \\
\hline Método de conteo & $\begin{array}{c}\text { Abundacia } \\
\text { asignada } \\
\text { subjetivamente }\end{array}$ & $\begin{array}{c}\text { Conteo e } \\
\text { identificación } \\
\text { de todos los } \\
\text { fragmentos } \\
\text { de cada } \\
\text { submuestra }\end{array}$ & $\begin{array}{c}\text { Método de } \\
\text { cuadrantes } \\
\text { y conteo de } \\
\text { hojas (de } \\
\text { Sphagnum) }\end{array}$ & $\begin{array}{l}\text { En cubeta de } \\
20 \times 10 \mathrm{~cm} \text { con } \\
\text { agua destilada. } \\
\text { Observación } \\
\text { bajo microscopio } \\
\text { estereoscopico } \\
\text { (10x y 50x) con } \\
\text { una cuadrícula de } \\
10 \times 10 \text { en ocular. } \\
\text { Restos estimados } \\
\text { como porcentajes } \\
\text { en } 15 \text { vistas al azar }\end{array}$ & $\begin{array}{c}\text { Bajo microscopio } \\
\text { esteroscopico } \\
\text { (20x), conteo } \\
\text { de todos los } \\
\text { fragmentos } \\
\text { presentes } \\
\text { en } 10 \mathrm{ml}\end{array}$ \\
\hline Repositorio & NE & $\begin{array}{l}\text { En glicerina. } \\
\text { Sphagnum, } \\
\text { en agua. } \\
\text { Preparados para } \\
\text { MO, en solucion } \\
\text { de Hoyer }\end{array}$ & NE & NE & $\begin{array}{c}\text { En } 75 \mathrm{~cm}^{3} \text { de } \\
\text { agua destilada } \\
\text { con } 2-3 \text { gotas de } \\
\mathrm{HCl} \text { al } 10 \% \text {, en } \\
\text { refrigerador } 4^{\circ} \mathrm{C} \\
+ \text { imagen digital }\end{array}$ \\
\hline
\end{tabular}

en el presente trabajo. Esto es más simple porque no debe sumergirse el material en agua y luego ser recuperado. A diferencia de la mayoría de trabajos previos, en el procesamiento propuesto no se realiza ningún tratamiento químico a la submuestra para evitar un posible daño del material (Beaudoin, 2007).

En cuanto al tamiz con el que se filtra el material a estudiar, en este trabajo se propone utilizar uno de tamaño de malla intermedio, así como fue utilizado por varios otros autores (Tabla 2 y Van der Putten et al., 2011). A su vez, a diferencia de aportes previos cómo el de Barber (1993) se prefiere un modo de tamizado menos agresivo, donde la muestra permanece en agua y se agita suavemente para lograr una disgregación sin desarticulación.

El método de conteo aquí propuesto resulta intermedio entre la propuesta exhaustiva de Janssens (1983) y la propuesta cualitativa de Walker \& Walker (1961). Debe mencionarse que en las submuestras donde Sphagnum es dominante difícilmente pueda contarse todo el material contenido en las cinco alícuotas en las cápsulas de Petri, porque en cada una es posible encontrar gran cantidad de fragmentos del mismo taxón (más de 500). Por lo que para esos casos se recomienda contar el contenido de una única alícuota. 


\section{A. Savoretti et al. - Metodología para macrorrestos de briofitas de turberas}

Otra recomendación importante en cuanto a la resolución en el conteo es no excluir (y separar) los macrorrestos de briofitas de aquellos de plantas vasculares e invertebrados. La presencia de éstos es importante en las comparaciones entre muestras e interpretaciones paleoambientales (Dickson, 1986). La incorporación de conteos de semillas y otros macrorrestos de plantas vasculares, exige la utilización de un tamaño de malla de 100 o $125 \mu \mathrm{m}$ (Mauquoy et al., 2010). A pesar de que el estudio de otros macrorrestos no sea el objetivo particular en una primera instancia, este procedimiento es mínimo comparado con el procesamiento de todo el testigo y facilita el trabajo posterior de los especialistas en los diferentes indicadores paleoambientales.

Finalmente, sólo en la metodología propuesta por Janssens (1983) se especifica qué tipo de material quedará como repositorio del muestro. En el método aquí propuesto, los macrorrestos se preservan de tres modos: preparados realizados para determinar los macrorrestos (luego material de referencia), el residuo de los $5 \mathrm{~cm}^{3}$ iniciales que no fue analizado del testigo original y que se almacena en agua destilada con $\mathrm{HCl}$ en refrigerador a $4^{\circ} \mathrm{C}$, y un resguardo en forma de imágenes digitales de cada submuestra general y sus cinco alícuotas.

\section{Conclusiones}

En el presente trabajo se presenta de manera detallada una nueva metodología para el procesamiento de testigos provenientes de turberas, con el fin de abordar estudios paleobrioflorístico. De la metodología aquí presentada vale destacar los siguientes aportes:

Disminución en el daño (dispersión y multiplicación) de los restos, en comparación con otras metodologías.

Incorporación del conteo de los diferentes tipos de fragmentos de cada uno de los taxones/ morfotipos con el objetivo de realizar estudios comparativos de la tafonomía y con ello enriquecer las interpretaciones paleoecológicas (por ejemplo, Tabla 1).

Incorporación del paso de digitalización de imágenes de las muestras, lo cual permite comparar de forma muy simple los macrorrestos entre alícuotas, así como entre secciones del testigo y entre testigos de turberas de cualquier región.
La estandarización de la digitalización permitiría el desarrollo de bases de datos de macrorrestos cuaternarios a nivel global.

\section{Agradecimientos}

Los autores agradecen a quienes contribuyeron en calidad de árbitros, con valiosos aportes para la mejora en la redacción y contenido del manuscrito. A Raúl Pózner por su colaboración durante el desarrollo de la metodología presentada.

\section{Bibliografía}

ANDERSON, L.E, J. A. SHAW \& B. SHAW. 2009. Peat mosses of Southeastern United State. New York Botanical Garden Press, New York.

BARBER, K. E. 1993.Peatlands as scientific archives of past biodiversity. Biodivers. \& Conservation 2: 474489.

BEAUDOIN, A. B. 2007. On the laboratory procedure for processing unconsolidated sediment samples to concentrate subfossil seed and other plant macroremains. Journal of Paleolimnology 37: 301308.

BIRKS, H. H. 2001. Plant macrofossils. En: SMOL, J. P., H. J. B. BIRKS \& W. M. LAST (eds.), Tracking environmental change using lake sediments. Volume 3: terrestrial, algal, and siliceous indicators, pp. 4974. Springer, Netherlands.

BIRKS, H. H. 2007. Plant macrofossil introduction. En: ELIAS, S. A. (ed.), Encyclopedia of Quaternary Science, vol. 3, pp. 2266-2288. Elsevier Scientific Publishing, Amsterdam.

BIRKS, H. H. 2017. Plant macrofossil introduction, Reference Module in Earth Systems and Environmental Sciences. Elsevier Scientific Publishing, Amsterdam.

CHAMBERS, F. M., S. A. BRAIN, D. MAUQUOY, J. MCCARROLL \& T. DALEY. 2014. The 'Little Ice Age' in the Southern Hemisphere in the context of the last 3000 years: Peat-based proxy-climate data from Tierra del Fuego. The Holocene 24: 1649-1656.

CLYMO, R.S. \& P.M. HAYWARD. 1982. The ecology of Sphagnum. En: SMITH, A. J. E. (ed.), Bryophyte Ecology, pp. 229-89. Chapman \& Hall, London.

DE VLEESCHOUWER F., F. M. CHAMBERS \& G. T. SWINDLES. 2010. Coring and sub-sampling of peatlands for palaeoenvironmental research. Mires and peat 7 .

DICKSON, J. H. 1986. Bryophyte analysis. En: BERGLUND, B. E. (ed.), Handbook of Palaeoecology and Palaeohydrology, pp. 627-643. Wiley, Chichester. 
HEUSSER, C. J. 1990. Late-glacial and Holocene vegetation and climate of subantarctic South America. Rev. Palaeobot. Palynol. 65, 9-15.

INGRAM, H .A. P. 1978. Soil layers in mires: function and terminology. Eur. J. Soil Sci. 29: 224-227.

ITURRASPE, R. 2010. Las turberas de Tierra del Fuego y el Cambio Climático global. Fundación Humedales, Buenos Aires, Argentina, 26 pp. Disponible en: http:/www.ambiente.gov.ar/archivos/web/GTRA/ file/Humedales/Turberas $\% 20 y \% 20$ CC.pdf [Acceso: 15 December 2016]

JAKAB, G., P. SÜMEGI \& E. MAGYARI. 2004. A new paleobotanical method for the description of Late Quaternary organic sediments (Mire -development pathaways and paleoclimatic records from $\mathrm{S}$ Hungary). Acta Geol. Hungarica 47: 373-409.

JANSSENS, J. A. 1983. A quantitative method for stratigraphic analysis of bryophytes in Holocene peat. J. Ecol. 71: 189-196.

JANSSENS, J. A. 1987. Ecology of peatland bryophytes and paleoenvironmental reconstruction of peatlands using fossil bryophytes. Manual for Bryiological Methods Workshop. Satellite Conference of the XIV. International Botanical Conference. International Association of Bryologists.

JANSSENS, J. A. 1988. Fossil bryophytes and paleoenvironmental reconstruction of peatlands. En: GLIME, J. M. (ed.), Methods in Bryology. Proceedings of the Bryological Methods Workshop, pp. 299-306. Mainz.

JANSSENS, J. A. 1990. Methods in Quaternary Ecology 11. Bryophytes. Geosci. Can. 17: 13-23.

LARRAÍN, J. 2015. Musgos y hepáticas en las turberas. En: DOMÍNGUEZ, E. \& D. VEGA-VALDÉS (eds.), Funciones y servicios ecosistémicos de las turberas en Magallanes. Colección de libros INIA $N^{\circ} 33$, pp. 127-145. Instituto de Investigaciones Agropecuarias. Centro Regional de Investigación Kampenaike. Punta Arenas, Chile.

MAUQUOY, D., M. BLAAUW, B. VAN GEEL, A. BORROMEI, M. QUATTROCCHIO, F. M. CHAMBERS \& G. POSSNERT. 2004. Late Holocene climatic changes in Tierra del Fuego based on multiproxy analyses of peat deposits. Quat. Res. 61: 148-158.

MAUQUOY, D., P. D. M. HUGHES \& B. VAN GEEL. 2010. A protocol for plant macrofossil analysis of peat deposits. Mires Peat 7: 1-5.

MARKGRAF, V. \& U. M. HUBER. 2010. Late and postglacial vegetation and fire history in Southern Patagonia and Tierra del Fuego. Palaeogeog. Palaeocl. 297: 351-366.

MILLER, N. G. 1980. Mosses as paleoecological indicators of late glacial terrestrial environments: some North American studies. B. Torrey Bot. Club 107: 373-391.
MILLER, N. G. 1983. Tertiary and Quaternary fossils. En: SCHUSTER R. M. (ed.), New Manual of Bryology, Volumen 1, Capítulo 20, pp. 1194-1232. The Hatori

OCHYRA, R., R. L. LEWIS SMITH \& H. BEDNAREK-OCHYRA. 2008. The illustrated moss flora of Antarctica. Cambridge University Press, Cambridge.

OCHYRA, R. \& C. M. MATTERI. 2001. Amblystegiaceae. En: GUARRERA, S. A., I. J. GAMUNDÍ DE AMOS \& D. R. DE HALPERÍN (eds.), Flora Criptogamica de Tierra del Fuego Volume XIV: Bryophyta, Musci, fascículo 10, pp. 1-96. CONICET, Buenos Aires.

SAVORETTI, A., M. S. CANDEL, M. FERNÁNDEZ, G. M. SUÁREZ, A. IGLESIAS \& J. F. PONCE. Late Glacial pioneer vegetation and paleoenvironments in the Beagle Channel, Tierra del Fuego, southernmost Argentina: preliminary results. En: DE ASSIS R. DOS SANTOS, F., J. S. DE NOVAIS \& L. C. L. E LIMA (eds.), Boletín de la Asociación Latinoamericana de Paleobotánica y Palinología, Número 16, p. 306. Instituto de Geociências, Universidade Federal do Rio Grande do Sul, Porto Alegre.

SCHOFIELD, W. B. 1985. Introduction to bryology. Blackburn Press, New Jersey.

VAN DER PUTTEN, N., H. STIEPARAERE, C. VERBRUGGEN \& C. OCHYRA. 2004. Holocene palaeoecology and climate history of South Georgia (sub-Antarctic) based on a macrofossil record of bryophytes and seeds. The Holocene 14:382-392.

VAN DER PUTTEN, N., J. P. HÉBRARD, C. VERBRUGGEN, B. VAN DE VIJVER, J. R. DISNAR, S. SPASSOV, D. KERAVIS, J. L. DE BEAULIEU, M. DE DAPPER, J. HUS, N. THOUVENY \& Y. FRENOT. 2008. An integrated palaeoenvironmental investigation of a 6200 year old peat sequence from Île de la Possession, Îles Crozet, sub-Antarctica. Palaeogeog. Palaeocl. 270: 179-195.

VAN DER PUTTEN, C. VERBRUGGEN, R. OCHYRA, J. L. DE BEAULIEU, M. DE DAPPER, N., S. SPASSOV, J. HUS \& N. THOUVENY. 2009. Peat bank growth, Holocene palaeoecology and climate history of South Georgia (sub-Antarctica), based on a botanical macrofossil record. Quaternary Sci. Rev. 28: 65-79.

VAN DER PUTTEN N., D. MAUQUOY, C. VERBRUGGEN \& S. BJÖRCK. 2011. Subantarctic peatlands and their potential as palaeoenvironmental and palaeoclimatic archives. Quatern. Int. 268: 65-76. 
A. Savoretti et al. - Metodología para macrorrestos de briofitas de turberas

VITT, D. H. \& M. LUTH. 2017. A Guide to mosses and liverworts of Alberta peatlands. Freiburg, Germany.

VITT, D. H. \& R. K. WIEDER. 2009. The structure and function of bryophyte-dominated peatlands. En: GOFFINET, B. \& SHAW, J. A. (eds.), Bryophyte Biology, pp. 357-392. Cambridge University Press, Cambridge.
WALKER, D. \& P. M. WALKER. 1961. Stratigraphic Evidence of Regeneration inSome Irish Bogs. Journal of Ecology 49:169-185.

Recibido el 1 de marzo de 2017, aceptado el 24 de mayo de 2017. 
